\title{
TA Anisotropy Summary
}

\author{
K. Kawata ${ }^{1, *}$, A. di Matteo ${ }^{2}$, T. Fujii ${ }^{1}$, D. Ivanov ${ }^{3}$, C.C.H. Jui ${ }^{3}$, J.P. Lundquist ${ }^{3}$, J.N. Matthews ${ }^{3}$, S. Ogio ${ }^{4}, H$. Sagawa $^{1}$, \\ G.B. Thomson ${ }^{3}, P$. Tinyakov ${ }^{2}, I$. Tkachev ${ }^{5}$, and $S$. Troitsky ${ }^{5}$ (On behalf of the Telescope Array Collaboration) \\ ${ }^{1}$ Institute for Cosmic Ray Research, University of Tokyo, Kashiwa, Chiba, Japan \\ ${ }^{2}$ Service de Physique Théorique, Université Libre de Bruxelles, Brussels, Belgium \\ ${ }^{3}$ High Energy Astrophysics Institute and Department of Physics and Astronomy, University of Utah, Salt Lake City, Utah, USA \\ ${ }^{4}$ Graduate School of Science, Osaka City University, Osaka, Osaka, Japan \\ ${ }^{5}$ Institute for Nuclear Research of the Russian Academy of Sciences, Moscow, Russia
}

\begin{abstract}
The Telescope Array (TA) is the largest ultra-high-energy cosmic-ray (UHECR) detector in the northern hemisphere. It consists of an array of 507 surface detectors (SD) covering a total $700 \mathrm{~km}^{2}$ and three fluorescence detector stations overlooking the SD array. In this proceedings, we summarize recent results on the search for directional anisotropy of UHECRs using the latest dataset collected by the TA SD array. We obtained hints of the anisotropy of the UHECRs in the northern sky from the various analyses.
\end{abstract}

\section{Introduction}

The observable sources of super-GZK ultra high energy cosmic ray (UHECR) events are thought to be limited to the local universe within distances of $\sim 100 \mathrm{Mpc}$ for proton/iron. In order to accelerate particles up to the ultrahigh-energy range, particles must be confined to the accelerator site for more than a million years by a strong magnetic field and/or a large-scale confinement volume [1]. This would thus limit the number of possible accelerators in the universe to astrophysical candidates such as galaxy clusters, supermassive black holes in active galactic nuclei (AGNs), jets and lobes of active galaxies, starburst galaxies, gamma-ray bursts, etc.. Extragalactic astrophysical objects form the well-known large-scale structure (LSS), most of which is spread along the "supergalactic plane" in the local universe.

The main difficulty in identifying the origin of UHECRs is the loss of directional information due to magnetic field induced deflection. The deflection angle of a $60 \mathrm{EeV}$ proton over a distance of $50 \mathrm{Mpc}$ is estimated to be a few degrees assuming models with an intergalactic magnetic field (IGMF) strength of $1 \mathrm{nG}$. Meanwhile, the estimated deflection by the galactic magnetic field (GMF) is between a few degrees and ten degrees, depending on the direction in the sky. If the highest-energy cosmic rays are protons coming from matter in the local universe such as the nearby galaxies, then the maximum amplitude of the cosmic-ray anisotropy above $\sim 60 \mathrm{EeV}$ is expected to be detectable by the latest UHECR detectors [2].

The largest UHECR detector in each hemisphere, Telescope Array (TA) in the north and Pierre Auger Observatory (Auger) in the south have both obtained some hints/evidence of anisotropy in cosmic-ray arrival direc-

*e-mail: kawata@icrr.u-tokyo.ac.jp tions. Recent anisotropy measurements and the efforts to identify UHECR sources are summarized in Ref. [3]. The Auger collaboration has found a dipole-like anisotropy with an amplitude of $6.5 \%$ for cosmic rays with energy greater than $8 \mathrm{EeV}$. The anisotropy has a statistical significance of $5 \sigma$ [4]. The Auger group also claimed a correlation between the flux pattern of nearby starburst galaxies and UHECRs with energy greater than $39 \mathrm{EeV}$. Here the statistical significance is at the $4 \sigma$ level [5]. Meanwhile, the TA collaboration has reported a cluster of UHECRs, with $E>57 \mathrm{EeV}$, in the first 5-years observation period from 2008 May to 2013 May [6]. This "hotspot" is centered near the Ursa Major cluster, and extends to $\sim 20^{\circ}$ angular scale. The chance probability of this hotspot in an isotropic cosmic-ray sky was calculated to be $3.7 \times 10^{-4}$ $(3.4 \sigma)$. Subsequently, the TA collaboration found evidence of intermediate-scale anisotropy in the energy spectrum above $10^{19.2} \mathrm{eV}$ in the TA hotspot direction using 7-year data [7].

In this proceeding, we summarize recent anisotropy studies made with data collected by the Telescope Array surface detector array.

\section{Telescope Array Experiment}

The Telescope Array (TA) is the largest cosmic-ray detector in the northern hemisphere. It consists of a surface detector (SD) array [8] and three fluorescence detector (FD) stations $[9,10]$. The TA has been fully operational in Millard Country, Utah, USA $\left(39.30^{\circ} \mathrm{N}, 112.91^{\circ} \mathrm{W}\right.$; about $1,400 \mathrm{~m}$ above sea level), since 2008. The TA SD array consists of 507 plastic scintillation detectors of $3 \mathrm{~m}^{2}$ area located on a square grid with $1.2 \mathrm{~km}$ separation and makes measurements of the footprints of extensive air showers when they arrive at the Earth's surface. The SD array has 
an area of approximately $700 \mathrm{~km}^{2}$. For more details, see Ref. [11]. The TA SD array observes cosmic rays from $\sim 1 \mathrm{EeV}$ to $100 \mathrm{EeV}$ using the extensive-air-shower technique of a scintillation detector array with a duty cycle near $100 \%$ regardless of weather conditions, and with a wide field of view. These capabilities give us an unbiased survey of the northern sky. The telescope stations observe the sky over the SD array and make measurements of the longitudinal development of the air showers as they traverse the atmosphere.

\section{Correlation with Large-Scale Structure}

The observable sources of super-GZK events are thought to be limited to the local universe within distances of $\sim 100 \mathrm{Mpc}$. Roughly, most types of extragalactic objects might be embedded in the large-scale structure (LSS) of the universe. Firstly, we compare the TA event distribution to the LSS hypothesis model based on the 2MASS Galaxy Redshift Catalog (XSCz), which represents the mass distribution in the Universe. In this model, we assume a proton composition and simulate the propagation from the sources to the Earth taking account of various energy losses. The simulated arrival directions are smeared by the 2D Gaussian with the angular width $\theta$ (smearing angle) as a free parameter, which represents the deflection angle of the UHECR in the magnetic fields. The final flux map is multiplied by the TA exposure to compare the observed arrival directions. For more details concerning the method, see Ref. [12]. The predicted flux model is then compared to the TA event distributions with the energy greater than 10, 40 and $57 \mathrm{EeV}$, respectively, while scanning the smearing angle. For comparison, we also test the TA events with the isotropic flux model.

Figure 1 shows the compatibility between TA events collected within 10 years of data and two models. The flux model is the LSS hypothesis (blue points) and the second is an isotropic hypothesis (green points). At the lower energies, $\mathrm{E}>10$ and $>40 \mathrm{EeV}$, the TA event distribution is consistent with an isotropic source distribution for any smearing angles, while the compatibility with the LSS hypothesis is poor at small smearing angles. However, at higher energies, $E>57 \mathrm{EeV}$, the LSS hypothesis agrees with the TA event distribution for moderate smearing angles $\left(10^{\circ}-25^{\circ}\right)$ while the data is incompatible with the isotropic model. In the next section, we will discuss the event distribution above $57 \mathrm{EeV}$ in the northern sky.

\section{Hotspot Above $57 \mathrm{EeV}$}

The Telescope Array collaboration reported on an indication of excess flux of UHECRs with E $>57 \mathrm{EeV}$ using 5year data [6]. This region of sky centered near the Ursa Major cluster was called the "hot-spot" and it extends to $\gtrsim 10^{\circ}$ angular scale. The chance probability finding a hotspot in an isotropic cosmic-ray sky was calculated to be $3.7 \times 10^{-4}(3.4 \sigma)$ assuming $15^{\circ}, 20^{\circ}, 25^{\circ}, 30^{\circ}$, and $35^{\circ}$ oversampling radius circles were searched.

In this work we test this result using the 10-year data collected by the TA SD array. We have observed 157
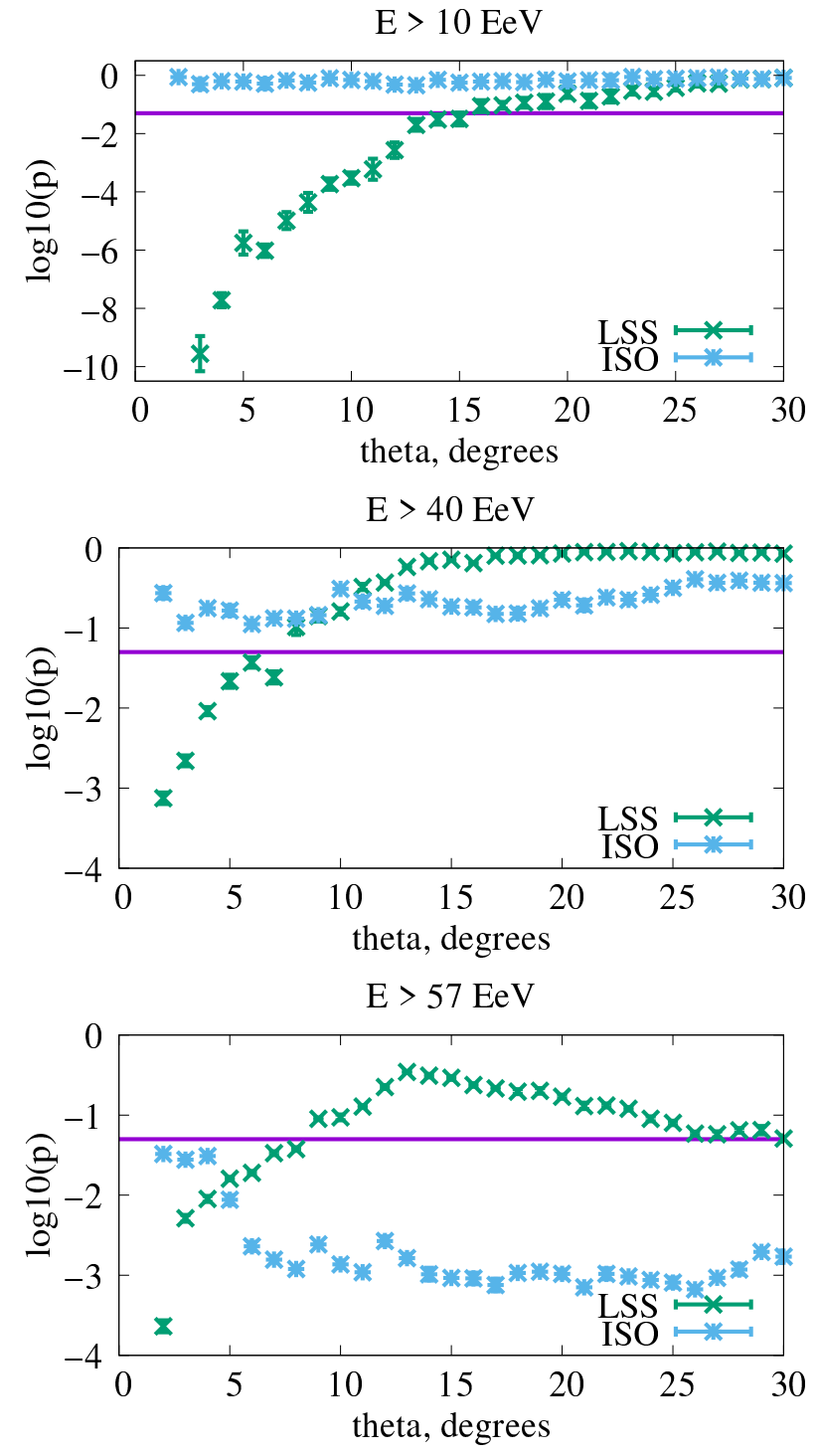

Figure 1. Compatibility between UHECR events observed by the TA for 10 years and two different models which are the flux model of the LSS hypothesis (blue points) and the isotropic hypothesis (green points). Three plots show compatibility for energy thresholds of $10 \mathrm{EeV}, 40 \mathrm{EeV}$, and $57 \mathrm{EeV}$, respectively. The horizontal red line indicates a $95 \%$ confidence level.

events with $E>57 \mathrm{EeV}$ in 10 years, which is approximately double statistics of the first 5-year observation. These events are summed over different five oversampling radius circles, $15^{\circ}, 20^{\circ}, 25^{\circ}, 30^{\circ}$, and $35^{\circ}$. The centers of tested directions are on a $0.1^{\circ} \times 0.1^{\circ}$ grid in the equatorial coordinates. We then search for the maximum significance over all grid points and five oversampling radius circles. We found the maximum significance of $5.0 \sigma$ at a position R.A. $=144.3^{\circ}$, and Dec. $=40.3^{\circ}$ with $25^{\circ}$ oversampling radius circle. The chance probability of the 10-year hotspot in an isotropic sky is estimated to be $2.1 \times 10^{-3}(2.9 \sigma)$. Figure 2 (right) shows the significance maps of the UHECR events with $\mathrm{E}>57 \mathrm{EeV}$ for 10 years with $25^{\circ}$ radius circle, compared with our previous result for the 1 st 5 years of data with $20^{\circ}$ (left) [6]. The 10-year hotspot looks larger (the number of background events in $25^{\circ}$ radius circle is $50 \%$ higher than that of $20^{\circ}$ radius circle), has extended all 

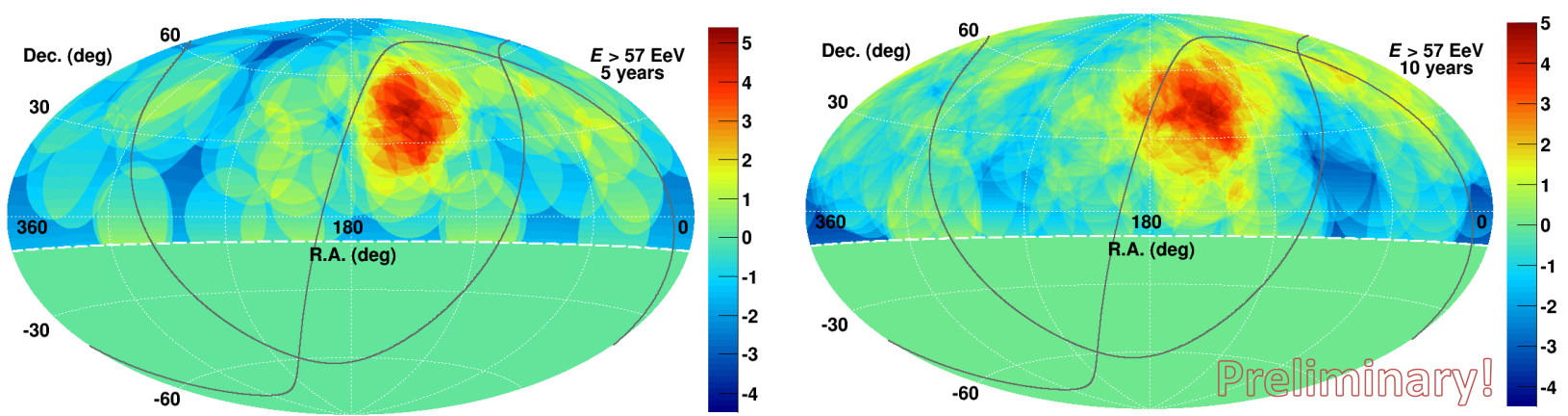

Figure 2. (Left) A significance map in the equatorial coordinates of arrival directions of UHECR events with $\mathrm{E}>57 \mathrm{EeV}$ for events observed in the 1 st 5 years of Telescope Array data. Events are smoothed by $20^{\circ}$ oversampling radius circle. (Right) A significance map of UHECR events with $\mathrm{E}>57 \mathrm{EeV}$ for the first 10 years of data. Here, the events are smoothed by $25^{\circ}$ oversampling radius circle which was determined to maximize the significance.

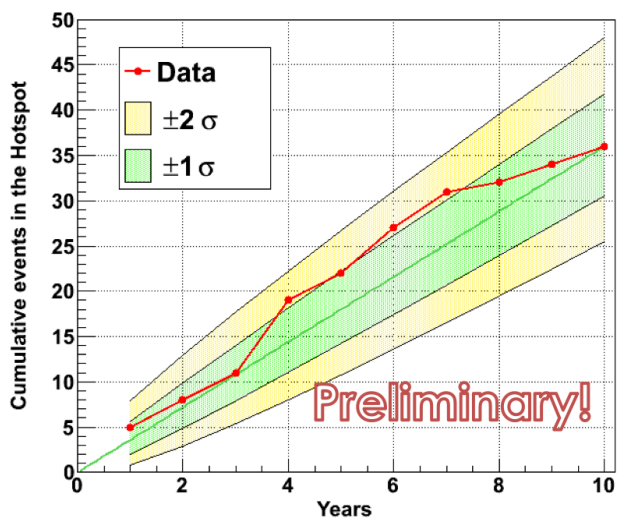

Figure 3. Integral time variation of the TA hotspot. Red points show the evolution of the data collection above $57 \mathrm{EeV}$ within the hotspot region. Green and yellow shaded areas show $1 \sigma$ and $2 \sigma$ deviations from the rate of data observation respectively, assuming a linear increase in rate.

the way to the SGP, and is irregular in shape. Therefore a circular oversampling shape is not really appropriate, and using one results in a lower limit on the significance. However, for simplicity we include such a calculation.

We divided the 10-year data into the first 5 years and the second 5 years. The significance at the hotspot position is $5.0 \sigma$ for the first 5 years and $2.0 \sigma$ for the second 5 years, respectively, with $25^{\circ}$ radius circle. Figure 3 shows the cumulative events inside the hotspot circle defined by the 10-year dataset to check a deviation from the linear increase. Green and yellow bands in this figure represent $1 \sigma$ and $2 \sigma$ allowed regions. The increase rate of the events inside the hotspot circle is consistent with a constant $2 \sigma$ excess.

\section{Spectral Anisotropy at the Hotspot}

We extend the hotspot study to lower energies, $\mathrm{E}<57 \mathrm{EeV}$ and search for specifically localized differences in the energy distribution of events. In this analysis, we compare the spectra inside and outside of the hotspot region [7].
See Figure 4 (Right). The difference between the spectra above $10^{19.2} \mathrm{eV}$ was evaluated by the Poisson likelihood ratio test. The position and size of the region that maximizes the spectral difference was allowed to differ from the previously described hotspot. A search therefore was performed by sliding a circular window over the sky, scanning the lower energy thresholds $\left(10^{19.0}, 10^{19.1}\right.$, $10^{19.2}$ and $10^{19.3} \mathrm{eV}$ ) in the spectrum. Figure 4 (Left) shows significance map of the spectral anisotropy above $10^{19.2} \mathrm{eV}$ which is smoothed by $14.03 \%$ equal exposure spherical cap bins. The maximum local significance in the whole sky is calculated to be $6.17 \sigma$, its position is located at R.A. $=9^{h} 6^{m}$, Dec. $=45^{\circ}$ which is near the center of the original hotspot direction. The flux with energies $10^{19.2}<E<10^{19.75} \mathrm{eV}$ has a deficit for and an excess for $\mathrm{E}>10^{19.75} \mathrm{eV}(\sim 57 \mathrm{EeV})$, respectively, compared with the average flux of the other sky. The post-trial probability of this spectral anisotropy appearing by chance from the isotropic distribution is estimated to be $9 \times 10^{-5}$, which corresponds to $3.74 \sigma$.

The spatial distribution of positions which have the spectral anisotropy in Fig. 4 seems to be parallelly shifted from the supergalactic plane as shown by the red curve. A shift from the supergalactic plane is estimated to be $-16.5^{\circ}$. It appears that an extended feature that could be correlated with supergalactic structure. Biermann et al. [13] argued that the possiblitity to focus the highest cosmic rays above $50 \mathrm{EeV}$ in the direction perpendicular to the supergalatic sheets. On the other hand Ryu et al. [14] discussed that lower energy cosmic rays transverse to cosmological sheets/filaments.

\section{Small-Scale Anisotropy Search Above $100 \mathrm{EeV}$}

The higher energy cosmic rays are expected to be more useful for identifying UHECR sources due to smaller magnetic deflections. The AGASA experiment found a few small-scale event clusters at the highest energy region in the northern sky [15]. We search for small-scale clusters of UHECRs with $\mathrm{E}>100 \mathrm{EeV}$ using the event list from the 10-year TA data and the 10-year Auger data, respec- 

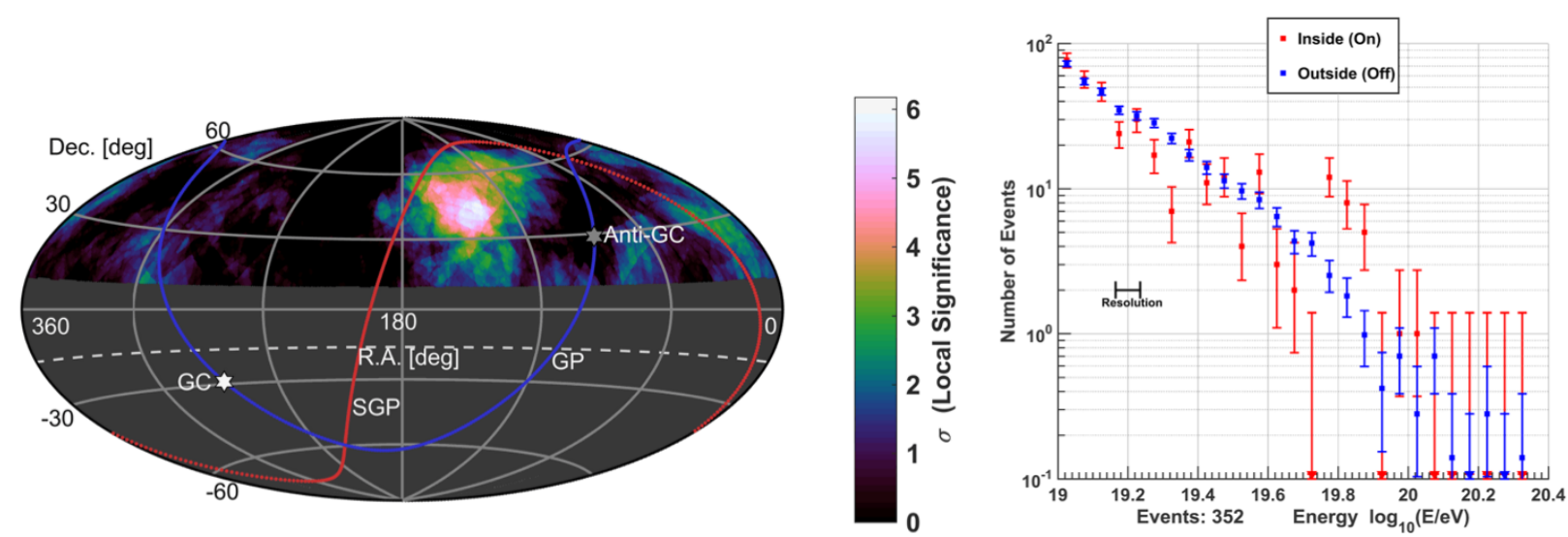

Figure 4. (Left) The significance map of the spectral anisotropy above $10^{19.2} \mathrm{eV}$ which is smoothed by $14.03 \%$ equal exposure spherical cap bins (from [7]). Solid curves indicate the galactic plane (GP) in blue and supergalactic plane (SGP) in red. The Galactic center (GC) is indicated by a white star and anti-galactic center (Anti-GC) by a gray star. (Right) The energy distribution inside the spherical cap bin (red) at the maximum significance position (R.A. $=9^{h} 16^{m}$, Dec. $\left.=45^{\circ}\right)$ in the direction of the Hotspot, compared to the expected distribution (blue).

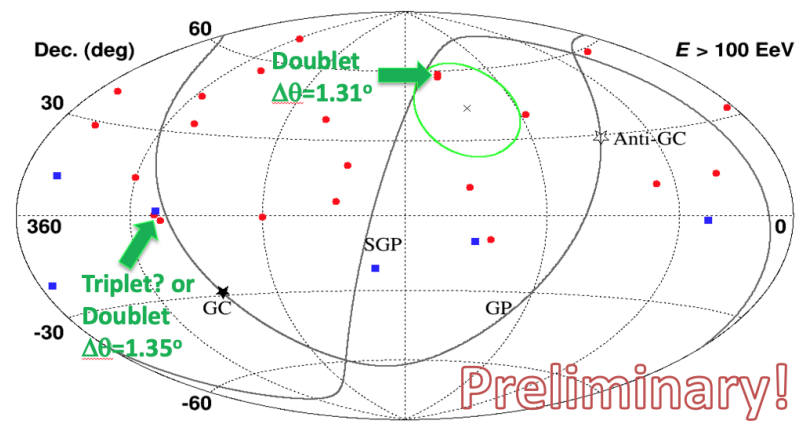

Figure 5. An Aitoff projection of the UHECR map in equatorial coordinates. The red circles and blue squares show directions of the UHECRs with E>100 EeV observed by the TA and the $\mathrm{PAO}$, respectively. The solid curves indicate the Galactic plane (GP) and the supergalactic plane (SGP). The closed and open stars indicate the Galactic center (GC) and the anti-Galactic center (Anti-GC).

tively. It is noted that the expected energy-scale difference between TA and Auger has not corrected here. Figure 5 shows the distribution of 28 cosmic-ray events E $>100 \mathrm{EeV}$ observed by the TA (red circles) and Auger (blue squares) in the equatorial coordinate. A doublet of events observed by TA and Auger near the equator has been reported by Troitsky [16]. In the updated data, we find a new event with $\mathrm{E}>100 \mathrm{EeV}$ near this doublet. Therefore, a triplet of events with $\mathrm{E}>100 \mathrm{EeV}$ is possible in the overlaping region of sky, although the angular distance between the doublet and this event is $3.7^{\circ}$, which can not be explained by our angular resolutions which are expected to be $\sim \sqrt{2^{\circ}}$ assuming neutral particles. It is noted that the deflection angle for $100 \mathrm{EeV}$ proton is expected to be a few degrees in Galactic magnetic field. In addition, we also find the another double of events with $\mathrm{E}>100 \mathrm{EeV}$ within the hotspot circle. The angular distance of this doublet is $1.31^{\circ}$, which is within our angular resolution $\sim \sqrt{2^{\circ}}$. Then, the chance probability of $\geq 2$ doublets at $<\sqrt{2^{\circ}}$ from the isotropic cosmic-ray sky is calculated to be $3 \times 10^{-3}(2.8 \sigma)$ by the Monte Carlo simulation.

\section{Correlation with Starburst Galaxy}

Recently, the Auger collaboration reported a correlation between the flux pattern of 23 nearby starburst galaxies (SBGs) and the arrival directions of the UHECR with E>39 EeV in the southern sky [5]. They investigated compatibilities between the observed arrival directions of the UHECRs and several flux pattern models based on the extragalactic source catalogs, such as the second catalog of hard Fermi-LAT sources (2FHL) and SBG list by the Fermi-LAT (Gamma Ray), and Swift-BAT (X-ray) and 2MASS (Optical) surveys. In order to evaluate the compatibility, they perform an unbinned maximum-likelihood method for each flux model. The test statistics (TS) value is derived from the likelihood ratio test between the flux model and an isotropic model. The best-fit parameters in each flux model are searched to maximize the TS value, scanning the parameter spaces, which are the threshold energy of observed events $\left(E_{\mathrm{th}}=1-80 \mathrm{EeV}\right)$, the ratio of the flux model to the isotropic flux $\left(f_{\text {ani }}\right)$, and the smearing angle of the model map $(\phi)$. They determined that the SBG flux model shows better compatibility than the isotropic model with a statistical significance $4.0 \sigma$, and the best-fit parameters for the SBG flux model are determined to be $E_{\text {th }}=39 \mathrm{EeV}, f_{\text {ani }}=9.7 \%$, and $\phi=12.9^{\circ}$.

Unlike the Auger's method, we do not scan the parameters to avoid any statistical penalty. We tested a SBG flux pattern using the Auger best-fit parameters, which are $E_{\mathrm{th}}=39 \mathrm{EeV}, f_{\mathrm{ani}}=9.7 \%$, and $\phi=12.9^{\circ}$. These were applied to the 9-year data collected by the Telescope Array [17]. It is noted that the energy loss of the UHECR is negligible in this model, because most of the flux come from within a few Mpc which are very nearby sources. The energy threshold is adopted to be $E_{\mathrm{th}}=43 \mathrm{EeV}$, cor- 

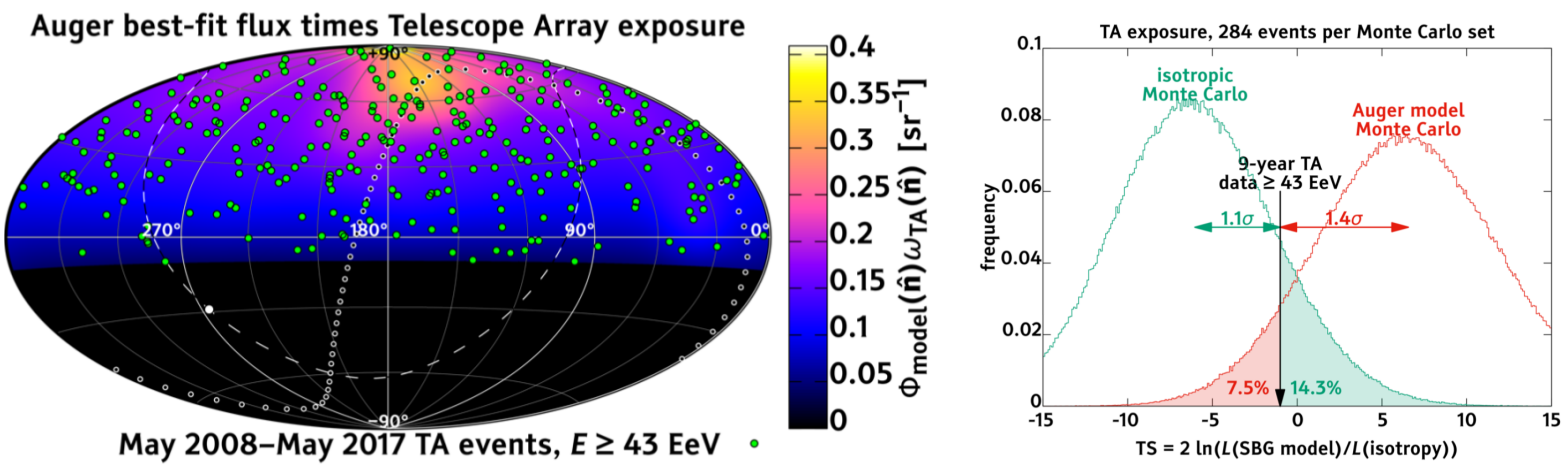

Figure 6. (Left) The green points show the event distribution observed by the TA during between May 2008 and May 2017, from [17]. The color contour shows the Auger best-fit flux model multiplied by the TA exposure. (Right) The TS distribution calculated by the MC simulation assuming the isotropic distribution (green) and the Auger SBG best-fit model (red). The downward arrow indicates the TS value estimated from the TA data.

responding to $39 \mathrm{EeV}$ in Auger when taking into account the energy-scale difference between two experiments. Figure 6 (Left) shows the TA event distribution (Points) overlapped with the Auger best-fit model map multiplied by the TA exposure (Contour). The TS value for this test is calculated to be -1.0 as shown in Fig. 6 (Right) by a downward arrow. In order to estimate chance probability of the obtained TS value, we generate a number of MC sets, each having 284 random directions which is same as statistics of the experimental data. The histograms in Fig. 6 (Right) represent the TS distribution by the MC sets assuming the isotropic distribution (green) and the Auger best-fit model (red). We find that the TA data is compatible with isotropy to within $1.1 \sigma$ and with the Auger result to within $1.4 \sigma$, meaning that it is not possible to discriminate between these two hypotheses. For more details, see Ref. [18].

\section{Supergalactic Structure of Multiplets}

If multiple UHECRs with different energies have traveled from a point source through the regular magnetic field, arrival directions could be aligned from the source direction in the sky. The angular distance of each event from the source direction should be inversely proportional to the energy. The lower energy events would be located away from the source and smeared by the random magnetic field, while the higher energy events would be placed close to the source direction with the smaller smearing angle. As a result, the multiple events are expected to be distributed within the wedge-like shape due to the regular and random magnetic fields. In this analysis, we investigate correlation between the cosmic-ray energy and the angular distance from a given direction in the wedge shape [19]. Previous similar studies of energy-position correlation did not yield results $[20,21]$. A wedge shape is defined by the pointing direction $(\phi)$, the wedge width $(\Delta \phi)$ and the maximum wedge distance $D$ (spherical cap radius).

In order to evaluate the correlation between the energy and angular distance in the wedge, we use Kendall's $\tau$ ranked correlation which measures the monotonic depen- dence between the variables [22].

$$
\tau=\frac{\text { (\# of concordant pairs })-(\# \text { of discordant pairs })}{n(n-1) / 2}
$$

Ranks are the ordering of the sorted variable (e.g. 1st, 2nd, etc.). A concordant pair of observations $\left(x_{\mathrm{i}}, y_{\mathrm{i}}\right)$ and $\left(x_{\mathrm{j}}, y_{\mathrm{j}}\right)$, where $i<j$, have equal differences in ranks $\left(x_{\mathrm{i}}>x_{\mathrm{j}}\right.$ and $y_{\mathrm{i}}>y_{\mathrm{j}}$ or the converse). They are discordant if $x_{\mathrm{i}}>x_{\mathrm{j}}$ and $y_{\mathrm{i}}<y_{\mathrm{j}}$ (or the converse). The correlation coefficient $\tau$ has a range from -1 to +1 , and the large $|\tau|$ indicates a strong correlation. Negative (positive) $\tau$ means energies are inversely-proportional (proportional) to angular distances in the wedge shape, while positive $\tau$ means energies are proportional to distances.

We search for the maximum $|\tau|$, scanning in 4 parameter spaces, which are $\phi, \Delta \phi, D$ and the energy threshold $E_{\mathrm{th}}$, in our FoV. Figure 7 (Left) shows the sky map of the maximum correlation strength $\tau$ in the supergalactic coordinate. The negative correlations of greater significance appear correlated with supergalactic plane. The post-trial significance is estimated to be greater than $\sim 4 \sigma$ by MC simulation. Figure 7 (Right) shows the mean $\tau$ inside equal solid angle bins for the data as a function of the supergalactic latitude. These results suggest that the higher energy events concentrate on the supergalactic plane, and a flow of magnetic deflection perpendicular to the supergalactic plane may exists [13].

\section{Summary}

In this proceeding, we have summarized recent results on the search for directional anisotropy of UHECRs using the data collected by the TA SD array as follows. For events with $\mathrm{E}>57 \mathrm{EeV}$, the TA event distribution is consistent with the LSS hypothesis at middle-scale smearing angles $\left(10^{\circ}-25^{\circ}\right)$, while the compatibility with the isotropic hypothesis is poor. The TA hotspot analysis was updated using the 10-year observation. The spectrum above $\mathrm{E}>10^{19.2} \mathrm{eV}$ around the TA hotspot seems to deviate from the average spectrum. Using the combined TA and Auger dataset above $100 \mathrm{EeV}$, we found 2 doublets within our 

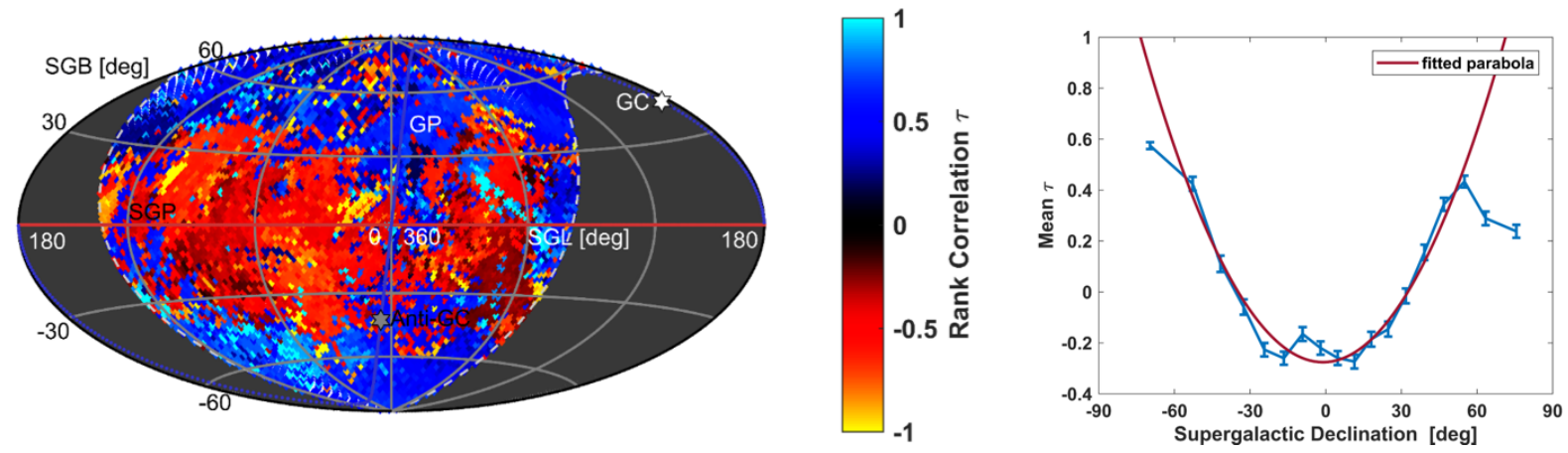

Figure 7. (Left) The sky map of the correlation strength $\tau$ in the supergalactic coordinate taken from [19]. The solid gray curve and red horizontal line indicate galactic plane (GP) and supergalactic plane (SGP), respectively. White and grey stars indicate the Galactic center (GC) and anti-galactic center (Anti-GC). (Right) The mean $\tau$ inside equal solid angle bins for the data as a function of the supergalactic latitude. The solid red curve shows the best-fit curve to the data by the parabola function $\left(a x^{2}+b x+c\right)$.

angular resolution. The chance probability of finding $\geq 2$ doublets is estimated to be $3 \times 10^{-3}$. We tested correlation between the TA events and the SBG flux model claimed by the Auger. Our result is consistent with both the isotropic model and the SBG flux model with Auger best-fit parameters so far. We also searched for UHECR multiplets which the correlation between the cosmic-ray energy and the angle distance from the vertex in the wedge shape. The strong correlations appear correlated with supergalactic plane. Thus, we obtained hints of the UHECR anisotropy in the northern sky with our current dataset. The TA will continuously observe to verify our current results. In addition, we are promoting the TA $\times 4$ project [23], which will extend the size of the TA SD by a factor of 4 , to collect data at a faster rate.

\section{Acknowledgments}

The Telescope Array experiment is supported by the Japan Society for the Promotion of Science(JSPS) through Grants-in-Aid for Priority Area 431, for Specially Promoted Research JP21000002, for Scientific Research (S) JP19104006, for Specially Promote Research JP15H05693, for Scientific Research (S) JP15H05741 and for Young Scientists (A) JPH26707011; by the joint research program of the Institute for Cosmic Ray Research (ICRR), The University of Tokyo; by the U.S. National Science Foundation awards PHY-0601915, PHY-1404495, PHY-1404502, and PHY-1607727; by the National Research Foundation of Korea (2015R1A2A1A01006870, 2015R1A2A1A15055344, 2016R1A5A1013277, 2016R1A2B4014967，2017R1A2A1A05071429); by the Russian Academy of Sciences, RFBR grant 1602-00962a (INR), IISN project No. 4.4502.13, and Belgian Science Policy under IUAP VII/37 (ULB). The foundations of Dr. Ezekiel R. and Edna Wattis Dumke, Willard L. Eccles, and George S. and Dolores Dore Eccles all helped with generous donations. The State of Utah supported the project through its Economic Development Board, and the University of Utah through the Office of the Vice President for Research. The experimental site became available through the cooperation of the Utah
School and Institutional Trust Lands Administration (SITLA), U.S. Bureau of Land Management (BLM), and the U.S. Air Force. We appreciate the assistance of the State of Utah and Fillmore offices of the BLM in crafting the Plan of Development for the site. Patrick Shea assisted the collaboration with valuable advice on a variety of topics. The people and the officials of Millard County, Utah have been a source of steadfast and warm support for our work which we greatly appreciate. We are indebted to the Millard County Road Department for their efforts to maintain and clear the roads which get us to our sites. We gratefully acknowledge the contribution from the technical staffs of our home institutions. An allocation of computer time from the Center for High Performance Computing at the University of Utah is gratefully acknowledged.

\section{References}

[1] Hillas, A. M. 1984, ARA\&A, 22, 425

[2] A. di Matteo and P. Tinyakov, MNRAS, 476, 715

[3] O. Deligny, K. Kawata and P. Tinyakov, PTEP., 12A104 (2017)

[4] A. Aab et al. (The Pierre Auger Collaboration), Science, 357, 1266 (2017)

[5] A. Aab et al. (The Pierre Auger Collaboration), ApJL, 853, L29 (2018)

[6] R. U. Abbasi et al. (The Telescope Array Collaboration), ApJL, 790, L21 (2014)

[7] R. U. Abbasi et al. (The Telescope Array Collaboration), ApJ 862, 91 (2018)

[8] H. Kawai et al.(The Telescope Array Collaboration), Nucl. Phys. B Proc. Suppl., 175, 221 (2008)

[9] H. Tokuno, et al. (The Telescope Array Collaboration), NIM-A, 676, 54 (2012)

[10] J. N. Matthews et al (The Telescope Array Collaboration), 30th ICRC (Merida), 5, 1157 (2007)

[11] T. Abu-Zayyad et al. (The Telescope Array Collaboration), NIM-A, 689, 87 (2012)

[12] T. Abu-Zayyad et al. (The Telescope Array Collaboration), ApJ, 757, 26 (2012) 
[13] P. L. Biermann, and H. Kang, and D. Ryu, arXiv:astro-ph/9709250 (1997)

[14] D. Ryu, H. Kang, and P. L. Biermann, A\&A, 335, 19 (1998)

[15] M. Takeda et al. (The AGASA Collaboration), ApJ 522, 225 (1999)

[16] S. V. Troitsky, JETP Letters, 96, 13 (2012)

[17] A. di Matteo, T. Fujii, and K. Kawata (The Telescope Array Collaboration), in this proceedings

[18] R. U. Abbasi et al. (The Telescope Array Collaboration), ApJL 867, L27 (2018)
[19] J. P. Landquist et al. (The Telescope Array Collaboration), in this proceedings

[20] P. Abreu et al. (The Pierre Auger Collaboration), Astropart. Phys., 35, 354 (2012)

[21] A. Aab et al. (The Pierre Auger Collaboration), Astropart. Phys., 68, 27 (2015)

[22] M. G. Kendall, Biometrika, 33, 239 (1945)

[23] E. Kido et al. (The Telescope Array Collaboration), in this proceedings 